\title{
PEGylated glucagon-like peptide-1 displays preserved effects on insulin release in isolated pancreatic islets and improved biological activity in $\mathrm{db} / \mathrm{db}$ mice
}

Received: 13 October 2005 / Accepted: 28 February 2006 / Published online: 4 April 2006

C) Springer-Verlag 2006

\begin{abstract}
Aims/hypothesis: The rapid degradation and clearance of glucagon-like peptide-1 (GLP-1) by the enzymes dipeptidyl peptidase-IV and neutral endopeptidase 24.11 are the main impediments to the development of GLP-1 as a potential glucose-lowering agent. In this study, new enzyme-resistant polyethylene glycol (PEG)conjugated GLP-1 analogues were designed and examined for metabolic stability and biological potency. Materials and methods: Two mono-PEGylated GLP-1 analogues, N-terminally modified N-PEG/GLP-1 and Lys-modified Lys-PEG/GLP-1, were prepared. Stability was tested in plasma and tissue extracts. In vitro insulin release studies were performed using isolated rat pancreatic islets, while in vivo glycaemic responses were measured in $d b / d b$ mice. Results: The half-life of Lys-PEG/GLP-1 was 40-, 10- and 28-fold longer than that of GLP-1 in plasma, liver and kidney homogenates, respectively. Lys-PEG/GLP-1 stimulated insulin secretion in the islets in a dose- and glucose-dependent manner, and was as potent as GLP-1. In contrast, N-PEG/GLP-1 showed extended metabolic stability but had significantly lower biological activity. The administration of Lys-PEG/GLP-1 ( $9 \mathrm{nmol} / \mathrm{kg}$ i.p.) to non-fasted $d b / d b$ mice stabilised glycaemia $(p<0.001)$, whereas GLP-1 (9 nmol/kg) only caused small changes in glucose level. During OGTT in fasted $d b / d b$ mice, LysPEG/GLP-1 administered at 1,3 and $9 \mathrm{nmol} / \mathrm{kg}$ (i.p.) reduced the glucose $\mathrm{AUC}_{0-3 \mathrm{~h}}$ by $48.7 \pm 9.4,55.0 \pm 2.9$ and $63.4 \pm 2.5 \%$, respectively, compared with placebo $(p<0.01)$,
\end{abstract}

S. Lee $\cdot$ Y. Byun

College of Pharmacy, Seoul National University,

San 56-1, Shillim-dong, Gwanak-gu,

Seoul 151-742, South Korea

Y. S. Youn · S.-H. Lee $\cdot$ K. C. Lee $(\bowtie)$

Drug Targeting Laboratory, College of Pharmacy,

SungKyunKwan University,

300 Chonchon-dong, Jangan-ku,

Suwon 440-746, South Korea

e-mail: kclee@skku.edu

Tel.: +82-31-2907704

Fax: +82-31-2907724 whereas GLP-1 ( $9 \mathrm{nmol} / \mathrm{kg})$ lowered the glucose level by $39.5 \pm 12.9 \%(p<0.01)$. Conclusions/interpretation: This study demonstrates that site-specific PEGylated GLP-1 analogues are resistant to degradation. The enhanced biological potencies of these analogues highlight their potential as new, GLP-1-like glucose-lowering agents.

Keywords GLP-1 - Metabolic stability · PEGylation • Pancreatic islets - Type 2 diabetes

Abbreviations DPP-IV: dipeptidyl peptidase-IV - GLP-1: glucagon-like peptide 1 - NEP: neutral endopeptidase PEG: polyethylene glycol

\section{Introduction}

Glucagon-like peptide-1 (7-36)amide (GLP-1) is a polypeptide hormone secreted from L cells in the gastrointestinal tract in response to the ingestion of nutrients [1]. GLP-1 is viewed as a potent therapy for type 2 diabetes $[2,3]$. However, its short circulating half-life means that high doses must be administered frequently, which limits its clinical application. The short half-live of GLP-1 is due to its rapid inactivation and clearance under physiological conditions by proteolytic enzymes such as dipeptidyl peptidase-IV (DPP-IV) [4] and neutral endopeptidase (NEP) 24.11 [5].

We recently proposed a novel and potent enzymeresistant form of a site-specific polyethylene glycolated (PEGylated) GLP-1 [6], and hypothesised that the covalent coupling of PEG to a specific site of GLP-1 would profoundly improve its enzymatic stability against DPP-IV and NEP 24.11, whilst retaining its biological activity. To this end, two series of mono-PEGylated GLP-1 analogues, N-terminally modified N-PEG/GLP-1 and Lys modified Lys-PEG/GLP-1, were prepared. These PEGylated GLP-1 analogues were clearly resistant to purified DPP-IV. Moreover, a pharmacokinetic evaluation in rats showed that the PEGylation process confers both an extended plasma half-life and a lower clearance rate, which result in 
a substantial improvement in the mean plasma residence time of GLP-1 after i.v. or s.c. administration.

In the present study we investigated the therapeutic potential of site-specific PEGylated GLP-1 by examining the impact of PEGylation on the biological activity and metabolic stability of GLP-1 in vitro, and its in vivo antihyperglycaemic effect in diabetic $d b / d b$ mice.

\section{Materials and methods}

Preparation of mono-PEGylated GLP-1 The PEG molecule was mono-substituted covalently at the N-terminus position $\mathrm{His}^{7}$ or at $\mathrm{Lys}^{34}$, and these analogues were designated N-PEG/GLP-1 and Lys-PEG/GLP-1, respectively [6].

Enzymatic stability in plasma and tissue homogenates The peptides were incubated at $37^{\circ} \mathrm{C}$ with the individual plasma and tissue homogenates, as described previously [7]. Half-life was estimated as the time (min) needed for the peptide concentration to be reduced by $50 \%$, as determined by calculating the peak area following reversed phase HPLC analysis.

Insulinotropic actions using isolated rat pancreatic islets Islets of Langerhans were isolated from male SpragueDawley rats, as described previously [8]. In vitro biological activity was evaluated by incubating 20 islets in $2 \mathrm{ml}$ of Krebs Ringer bicarbonate HEPES buffer (containing $16.7 \mathrm{mmol} / 1$ glucose) containing the respective stimuli at various concentrations for $2 \mathrm{~h}$ at $37^{\circ} \mathrm{C}$ in an atmosphere of $95 \%$ air, $5 \% \mathrm{CO}_{2}$. We carried out a separate set of experiments, incubating islets in the presence of $2.8,5.5$, 11.1 and $16.7 \mathrm{mmol} / 1$ glucose and $10 \mathrm{nmol} / \mathrm{l}$ of the respective peptides. Levels of insulin release were measured using radioimmunoassay kits (Insulin Kit; ICN Pharmaceuticals, Orangeburg, NY, USA).

Animals The animals were cared for according to the National Institutes of Health (NIH) guidelines for the care and use of laboratory animals (NIH publication 85-23, revised 1985). C57BL/6 $d b / d b$ female mice (7-10 weeks old; from the Korea Research Institute of Bioscience and Biotechnology, Daejon, Korea) were used. In order to determine the biological potency of the peptides, nonfasted diabetic rodents were given a single i.p. injection of $9 \mathrm{nmol} / \mathrm{kg}$ of the peptides. Blood samples were collected from a tail vein, and blood glucose levels were determined using a one-touch blood glucose monitoring system (Glucocard II; Arkray, Kyoto, Japan).

OGTT Overnight-fasted diabetic mice received an i.p. injection of either saline or one of the peptides. At time $-30 \mathrm{~min}$, saline or 1,3 or $9 \mathrm{nmol} / \mathrm{kg}$ of the peptides were administered i.p. At $0 \mathrm{~min}, 1.5 \mathrm{~g} / \mathrm{kg}$ of glucose in PBS $(10 \mathrm{mmol} / \mathrm{l}, \mathrm{pH} 7.4)$ was given orally to each group $(n=6)$. Blood glucose was monitored as described above.
Data analysis Data are expressed as means $\pm \mathrm{SD}$ in the text and as means \pm SEM in the figures. The Mann-Whitney non-parametric test or the $t$-test were used, depending on variances, to assess the statistical significance of differences. A $p$ value of $<0.05$ was considered significant.

\section{Results}

Proteolytic stabilities GLP-1 was rapidly degraded in rat plasma, liver and kidney homogenates in vitro $\left(t_{1 / 2}\right.$ values of $114 \pm 28,6 \pm 0.5,1 \pm 0.2 \mathrm{~min}$, respectively). In contrast, Lys-PEG/GLP-1 and N-PEG/GLP-1 were found to have significantly longer half-lives than native GLP-1, with $t_{1 / 2}$ values in plasma, liver and kidney homogenates of $4471 \pm$ $1822,62 \pm 23$ and $28 \pm 1 \mathrm{~min}$ for Lys-PEG/GLP-1, and $7560 \pm$ $5635,250 \pm 117$ and $23 \pm 7$ min for N-PEG/GLP-1, respectively $(p<0.01$ vs GLP-1).

In vitro biological activities In order to determine in vitro dose-response curves in terms of insulin released by GLP-1 and PEGylated GLP-1 analogues, peptide concentrations in media containing $16.7 \mathrm{mmol} / 1$ glucose were varied from 0.1-100 nmol/1 (Fig. 1a). GLP-1 and LysPEG/GLP-1 showed dose-dependent responses. Furthermore, at concentrations of $100 \mathrm{nmol} / \mathrm{l}$, Lys-PEG/GLP-1 proved as effective as GLP-1 at stimulating insulin secretion $\left(354.6 \pm 59.0\right.$ vs $312.2 \pm 63.7[\mathrm{pmol} / 1]$ islet $^{-1} \mathrm{~h}^{-1}$, respectively, $p=0.19$ ) and was significantly more potent than N-PEG/GLP-1, which showed a 1.7 \pm 0.6 -fold increase in insulin secretion per islet compared with the control, which contained no peptide.

Islets were cultured in different glucose concentrations $(2.8,5.5,11.1$ and $16.7 \mathrm{mmol} / \mathrm{l})$ with or without the peptides $(10 \mathrm{nmol} / \mathrm{l})$ to determine if PEGylated GLP-1 could retain its glucose-dependent insulinotropic profile (Fig. 1b). Islets cultured in 11.1 and $16.7 \mathrm{mmol} / \mathrm{l}$ of glucose showed rates of insulin secretion $4.0 \pm 1.5$ and $8.2 \pm$ 1.3 times higher than those observed in islets cultured in basal glucose $(2.8 \mathrm{mmol} / \mathrm{l})$, respectively. The addition of GLP-1 (10 nmol/l) further enhanced the level of insulin release by $14.8 \pm 2.8$-fold and $21.8 \pm 5.2$-fold at 11.1 and $16.7 \mathrm{mmol} / 1$ glucose, respectively. Lys-PEG/GLP-1 showed insulin secretion patterns that were comparable to those of native GLP-1 (increases of $11.0 \pm 2.2$ and $17.1 \pm$ 5.8 -fold at 11.1 and $16.7 \mathrm{mmol} / 1$ glucose, respectively). N-PEG/GLP-1 exhibited a lower biological activity.

Glucodynamic profiles in vivo To investigate the biological potency of PEGylated GLP-1 in vivo, equivalent doses $(9 \mathrm{nmol} / \mathrm{kg}$ ) of the peptides were administered to $d b / d b$ mice by i.p. injection (Fig. 2a). Blood glucose reduced significantly after the administration of Lys-PEG/GLP-1 as compared with GLP-1 or saline. Furthermore, Lys-PEG/ GLP-1 reduced basal glycaemia by $59.0 \pm 10.5 \%$ within $2 \mathrm{~h}$ $(10.4 \pm 2.7$ vs $25.2 \pm 5.9 \mathrm{mmol} / 1$ for saline, $p<0.01)$, and glycaemia remained stabilised for up to $5 \mathrm{~h}$ post-injection. The glucose $\mathrm{AUC}_{0-5 \mathrm{~h}}$ for Lys-PEG/GLP-1 was also significantly $(p<0.001)$ reduced compared with that for 

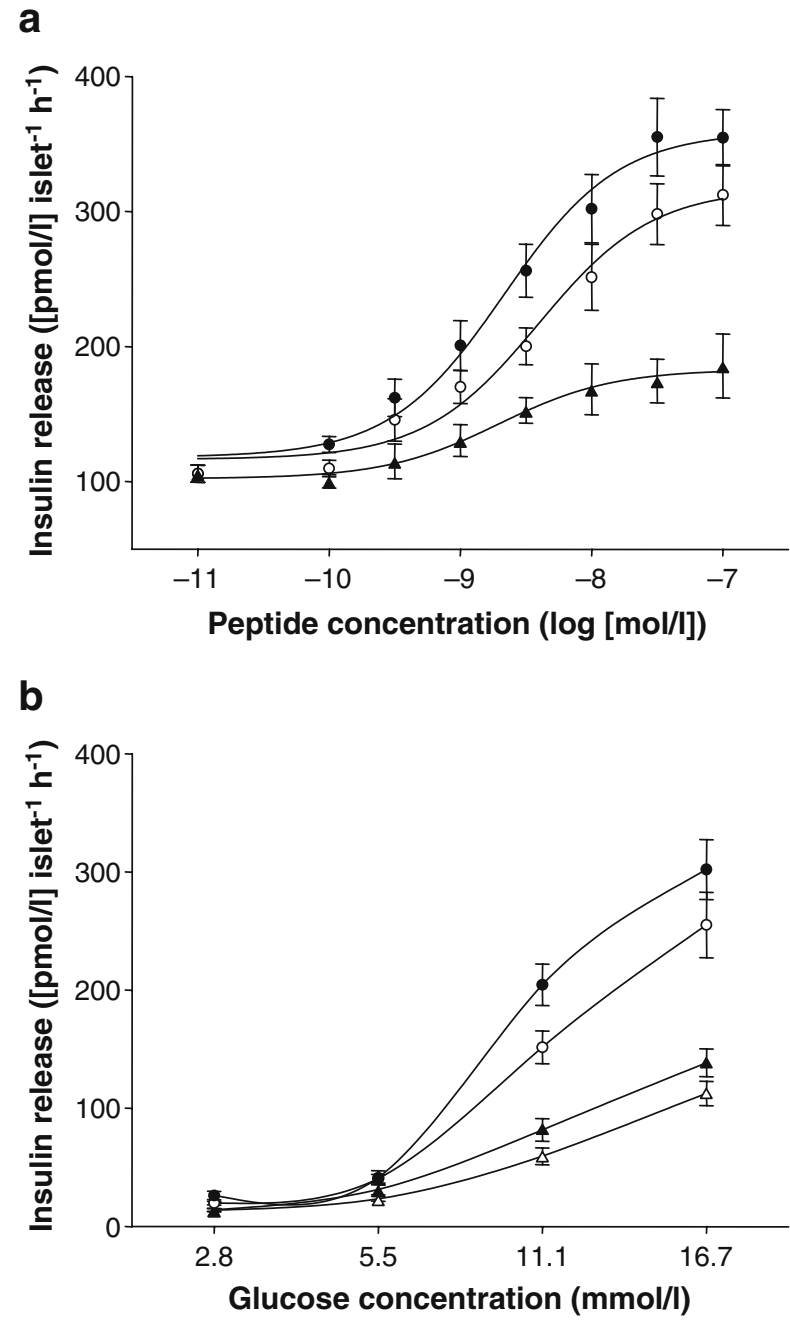

Fig. 1 In vitro biological activities of PEGylated GLP-1 analogues. a Stimulation of insulin secretion from isolated rat pancreatic islets in the presence of various doses of GLP-1 (filled circles), Lys-PEG/ GLP-1 (empty circles) or N-PEG/GLP-1 (filled triangles). Rat islets were isolated and then statically incubated (20 islets per sample) for $2 \mathrm{~h}$ at $37^{\circ} \mathrm{C}$ in the presence of $16.7 \mathrm{mmol} / 1$ glucose. Insulin release was measured by RIA. b Insulin secretion stimulation from isolated rat pancreatic islets by $10 \mathrm{nmol} / 1$ of GLP-1, Lys-PEG/GLP-1 or N-PEG/GLP-1, or without peptide (empty triangles) in the presence of various glucose concentrations for $2 \mathrm{~h}$ at $37^{\circ} \mathrm{C}$. Results are presented as means \pm SEM $(n=6)$

saline, whereas GLP-1 only induced small changes (glucose $\mathrm{AUC}_{0-5} \mathrm{~h}$ values for saline, GLP-1, Lys-PEG/ GLP-1 and N-PEG/GLP-1 were $109.7 \pm 28.5,95.0 \pm 13.2$, $53.4 \pm 13.7$, and $101.2 \pm 12.6[\mathrm{mmol} / 1] \mathrm{h}^{-1}$, respectively).

Dose-response relationships between GLP-1 or PEGylated GLP-1 and glucose tolerance were investigated (Fig. 2b). The administration of GLP-1 at a dose of 1 or $3 \mathrm{nmol} / \mathrm{kg}$ did not appreciably improve glucose tolerance. However, GLP-1 at a dose of $9 \mathrm{nmol} / \mathrm{kg}$ showed enhanced glucose elimination. This was evident from reductions in the glucose level and the glucose $\mathrm{AUC}_{0-3 \mathrm{~h}}(42.1 \pm 5.8$ $(p<0.05)$ and $37.0 \pm 7.9[\mathrm{mmol} / 1] \mathrm{h}^{-1}(p<0.01)$, for 3 and $9 \mathrm{nmol} / \mathrm{kg}$ of GLP-1, respectively, vs $55.2 \pm 10.1[\mathrm{mmol} / \mathrm{l}]$ $\mathrm{h}^{-1}$ for saline). In contrast, Lys-PEG/GLP-1 markedly a

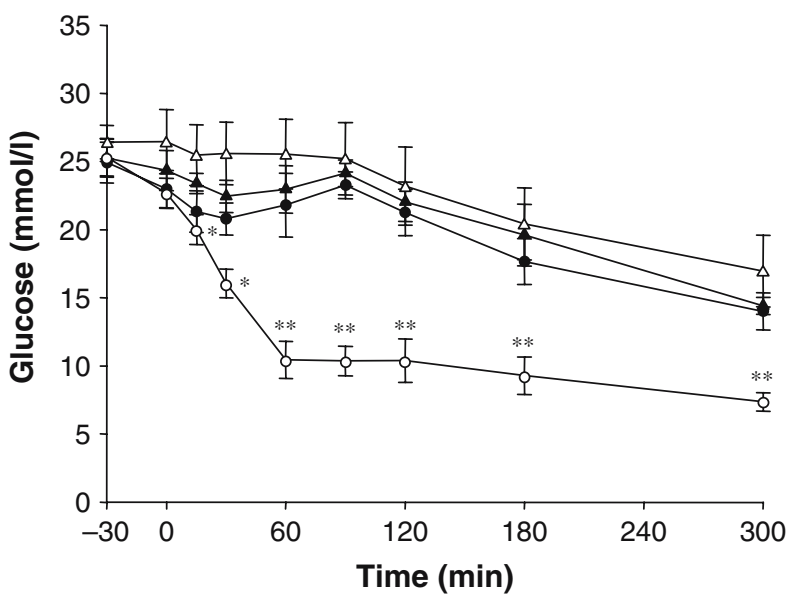

b

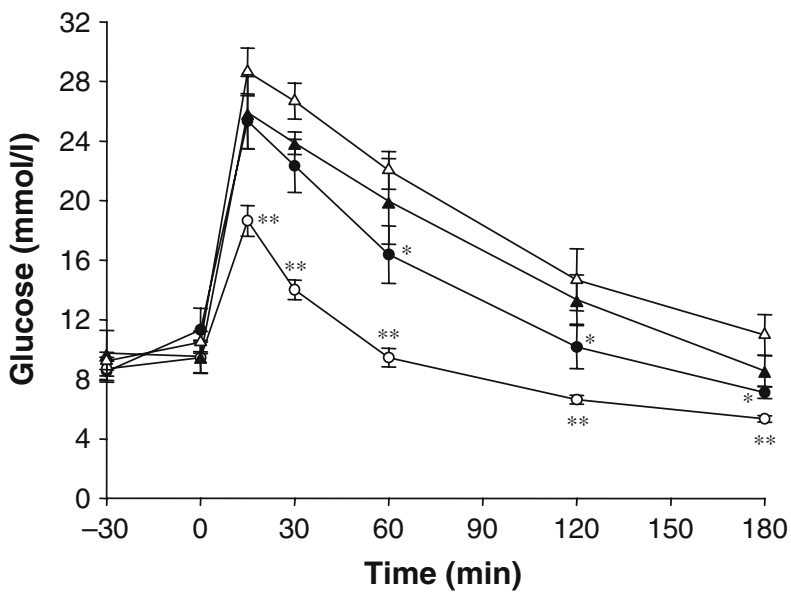

Fig. 2 Effect of a single i.p. injection of saline (empty triangles), GLP-1 (filled circles), Lys-PEG/GLP-1 (empty circles) or N-PEG/ GLP-1 (filled triangles) in female $d b / d b$ mice. a Blood glucose levels in fed $d b / d b$ mice after an i.p. injection of saline or peptide $(9 \mathrm{nmol} / \mathrm{kg}$ ). b Blood glucose levels in overnight-fasted $d b / d b$ mice during an oral glucose tolerance test $(1.5 \mathrm{~g} / \mathrm{kg})$ performed $30 \mathrm{~min}$ after an i.p. injection of saline or the administration of $3 \mathrm{nmol} / \mathrm{kg}$ of peptide. Results are presented as means $\pm \operatorname{SEM}(n=6) .{ }^{?} p<0.05$ and $p<0.01$ vs saline-treated control mice

improved glucose tolerance, with significantly lower blood glucose levels achieved throughout the study period compared with saline and with native GLP-1. When LysPEG/GLP-1 (1, 3 or $9 \mathrm{nmol} / \mathrm{kg})$ was administered, significant dose-dependent reductions in the peak blood glucose level and the glucose $\mathrm{AUC}_{0-3 \mathrm{~h}}$ were observed $\left(31.3 \pm 5.7,27.5 \pm 1.8\right.$ and $22.3 \pm 1.5[\mathrm{mmol} / 1] \mathrm{h}^{-1}$, respectively, $p<0.01$ vs saline). The administration of N-PEG/ GLP-1 did not alter blood glucose levels significantly.

\section{Discussion}

In this study we investigated the metabolic stability, biological activity and the acute effects of site-specific PEGylated Lys-PEG/GLP-1 as a potential replacement for GLP-1. We first investigated the impact of PEGylation on 
the metabolic stability of GPL-1 in vitro. It was hypothesised that conjugation of inert polymeric water-soluble substances on peptide surfaces could sterically hinder the approach of proteolytic enzymes [9], and that this would substantially improve in vivo stability. In the present study, compared with GLP-1, N-PEG/GLP-1 and Lys-PEG/GLP-1 showed much longer half-lives in plasma and in liver and kidney homogenates.

The biological activity of PEGylated GLP-1 analogues was investigated in vitro using pancreatic islets. Lys-PEG/ GLP-1 was almost as potent at stimulating insulin secretion as GLP-1, and did so in a dose- and glucose-dependent manner. These findings suggest that the PEG molecule at lysine effectively protects GLP-1 from proteolytic enzyme attack without altering its biological activity. In contrast, N-PEG/GLP-1 showed improved stability but significantly lower biological activity compared with Lys-PEG/GLP-1.

In order to determine if the PEGylated GLP-1 analogues are potential treatments for type 2 diabetes, we evaluated the acute antihyperglycaemic effects by administering them by i.p. injection to diabetic $d b / d b$ mice. Our findings show that Lys-PEG/GLP-1 produced the desired biological effect in a dose-dependent manner. Furthermore, Lys-PEG/ GLP-1 significantly reduced peak blood glucose levels after OGTT. This finding is further substantiated by our previous pharmacokinetic results in rats [6]. Taken with our enzyme-resistance results, Lys-PEG/GLP-1 shows greater longevity than GLP-1 in plasma under physiological conditions. The combination of augmented beta cell insulin secretion, increased insulin sensitivity (i.e. by OGTT) and improved half-life in plasma explain the durable glucoselowering effect of Lys-PEG/GLP-1. From this point of view, Lys-PEG/GLP-1 fulfilled all the features expected of a PEGylated GLP-1.

This study demonstrates that site-specific PEGylated GLP-1 analogues are resistant to degradation. In addition, their enhanced biological potencies highlight their potential as new GLP-1-like glucose-lowering agents.

Acknowledgement This study was supported by a grant from the Korea Health 21 R\&D Project, Ministry of Health \& Welfare, Republic of Korea (A050311).

Duality of interest The authors are not aware of any duality of interest.

\section{References}

1. Kieffer TJ, Habener JF (1999) The glucagon-like peptides. Endocr Rev 20:876-913

2. Deacon CF (2004) Therapeutic strategies based on glucagonlike peptide 1. Diabetes 53:2181-2189

3. Holst JJ, Orskov C (2004) The incretin approach for diabetes treatment. Diabetes 53:S197-S204

4. Kieffer TJ, McIntosh CH, Pederson RA (1995) Degradation of glucose-dependent insulinotropic polypeptide and truncated glucagon-like peptide 1 in vitro and in vivo by dipeptidyl peptidase IV. Endocrinology 136:3585-3596

5. Plamboeck A, Holst JJ, Carr RD, Deacon CF (2005) Neutral endopeptidase 24.11 and dipeptidyl peptidase IV are both mediators of the degradation of glucagon-like peptide 1 in the anaesthetised pig. Diabetologia 48:1882-1890

6. Lee SH, Lee S, Youn YS et al (2005) Synthesis, characterization, and pharmacokinetic studies of PEGylated glucagon-like peptide-1. Bioconjug Chem 16:377-382

7. Na DH, Youn YS, Park EJ et al (2004) Stability of PEGylated salmon calcitonin in nasal mucosa. J Pharm Sci 93:256-261

8. Lacy PE, Kostianovsky M (1967) Method for the isolation of intact islets of Langerhans from the rat pancreas. Diabetes 16:35-39

9. Harris JM, Chess RB (2003) Effect of pegylation on pharmaceuticals. Nat Rev Drug Discovery 2:214-221 